\title{
IMPURITY DIFFUSION COEFFICIENTS OF AL AND ZN IN MG DETERMINED FROM SOLID-TO- SOLID DIFFUSION COUPLES
}

\author{
Catherine Kammerer ${ }^{1}$, Nagraj Kulkarni ${ }^{2}$, Robert Warmack ${ }^{2}$, Kelly Perry ${ }^{2}$ \\ Irina Belova ${ }^{3}$, Graeme Murch ${ }^{3}$, Yongho Sohn ${ }^{1}$ \\ ${ }^{1}$ University of Central Florida, Advanced Materials Processing and Analysis Center \\ Department of Materials Science and Engineering, Orlando, FL, 32816, USA \\ ${ }^{2}$ Oak Ridge National Laboratory, Measurement Science \& Systems Engineering Division \\ Oak Ridge, TN, 37831, USA \\ ${ }^{3}$ The University of Newcastle, Center for Mass and Thermal Transport in Engineering Materials \\ School of Engineering, Callaghan, NSW 2308, Australia
}

Keywords: Magnesium, Aluminum, Zinc Interdiffusion, Impurity Diffusion, Hall Method

\begin{abstract}
Increasing use and development of lightweight $\mathrm{Mg}$ alloys have led to the desire for more fundamental research in and understanding of Mg-based systems. As property enhancing components, $\mathrm{Al}$ and $\mathrm{Zn}$ are two of the most important and common alloying elements for $\mathrm{Mg}$-alloys. We have investigated the concentration dependent interdiffusion of $\mathrm{Al}$ and $\mathrm{Zn}$ in $\mathrm{Mg}$ using diffusion couples of pure polycrystalline $\mathrm{Mg}$ mated to $\mathrm{Mg}$ solid solutions containing either $<9$ at.\% $\mathrm{Al}$ or $<3$ at. $\% \mathrm{Zn}$. Concentration profiles were determined by electron micro-probe microanalysis of the diffusion zone. The interdiffusion coefficients were determined by the classical Boltzmann-Matano method within the $\mathrm{Mg}$ solid solution. As the concentration of $\mathrm{Al}$ or $\mathrm{Zn}$ approaches the dilute ends, we employ an analytical approach based on the Hall method to estimate the impurity diffusion coefficients. Results of $\mathrm{Al}$ and $\mathrm{Zn}$ impurity diffusion in $\mathrm{Mg}$ are reported and compared to published impurity diffusion coefficients typically determined by thin film techniques.
\end{abstract}

\section{Introduction}

Integration of wrought magnesium alloys into automotive applications is intended to improve fuelefficiency and thereby reduce emissions. While magnesium is abundant and lightweight, its poor cold forming properties and low ductility, limited hightemperature properties, and poor corrosion resistance have mandated alloy development and thus fundamental research [1-5]. Two of the most common alloying elements in magnesium alloys are aluminum and zinc. Aluminum (FCC) and zinc (HCP) are relatively soluble in magnesium (HCP), but their solubility decreases at low temperatures. Aluminum additions yield alloys with a good balance between strength and ductility and are age hardenable with the precipitation of $\mathrm{Mg}_{17} \mathrm{Al}_{12}$. Similarly, zinc is added, often times with aluminum, to magnesium in order to improve room temperature properties and corrosion resistance. Magnesium alloyed with zinc can be heat treated to form MgZn precipitates. Diffusion plays a key role in the kinetics of many microstructural changes that occur during processing of magnesium alloys. Therefore, in this study, we examined the impurity diffusion of $\mathrm{Al}$ and $\mathrm{Zn}$ using solid-to-solid diffusion couples. Concentration profiles were determined by electron micro-probe microanalysis of the diffusion zone. The interdiffusion coefficients were determined by the classical Boltzmann-Matano method within the $\mathrm{Mg}$ solid solution. However, the Boltzmann-Matano method has a large margin of error for infinitely dilute compositions thus an analytical approach based on the Hall method is used to estimate the impurity diffusion coefficients.

\section{Experimental Procedure}

The magnesium alloys, Mg-9wt.\% Al (MA9) and Mg6wt.\%Zn (MZ6) were produced by Magnesium Elektron, North America. The pure $\mathrm{Mg}$ (Polycrystalline, 99.9\%) was commercially procured from Alfa Aesar. The alloys were direct chill cast, homogenized, and then warm extruded. All material was subjected to a grain-growth anneal roughly 40 $50^{\circ} \mathrm{C}$ below solidus for $8-16$ hours. The grain size was determined be between $100-500 \mu \mathrm{m}$. The material was then sectioned into $3-4 \mathrm{~mm}$ thick discs. The disc specimens were polished to a $1 \mu \mathrm{m}$ surface finish using a non-oxidizing lubricant. The diffusion couples, Mg vs. MA9 and Mg vs. MZ6, were then assembled with 2 mm-thick $\mathrm{Al}_{2} \mathrm{O}_{3}$ spacers in stainless steel jigs as shown in Figure 1. 


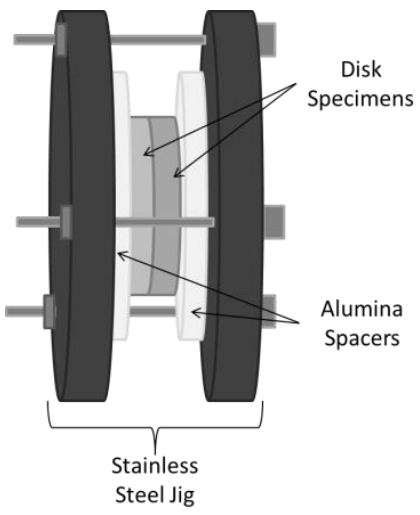

Figure 1: Schematic representation of a solid-to-solid diffusion couple assembly.

The jig assemblies were encapsulated individually in quartz capsules, flushed with argon and hydrogen, and then evacuated to $10^{-6}$ Torr. The encapsulated assemblies were placed in a Paragon Bluebird ${ }^{\mathrm{TM}}$ furnace that was preheated to the annealing temperature. The temperature of the diffusion couples was monitored with an independent resistance temperature detector probe with an Omega ${ }^{\mathrm{TM}}$ data acquisition system. Diffusion anneal times and temperatures are presented in Table 1. After the annealing cycle the capsules were quickly removed from the furnace and quenched in water. The couple was extracted from the jig and mounted in epoxy. Once cured, the couple was cross-sectioned and metallographically prepared down to $1 \mu \mathrm{m}$ finish, again using a non-oxidizing lubricant. Each diffusion couple was examined using optical microscopy first to check the diffusion bond integrity, then using Scanning Electron Microscope equipped with an Xray Energy Dispersive Spectrometer (Zeiss Ultra 55 SEM with EDS) to quantify the thickness of the interdiffusion zone (IZ). Further studies carried out also employed electron probe microanalysis (EPMA) (JEOL JXA-8200) for the determination of concentration profiles. Each couple was interrogated by the EPMA. Fitted concentration profiles were extracted from the EPMA data using either a cubic smoothing spline or a Savitzky-Golay smoothing function. The fitted profiles were then used in the determination of composition-dependent interdiffusion coefficients in $\mathrm{Mg}(\mathrm{Al})$ - and $\mathrm{Mg}(\mathrm{Zn})$ solid solutions, calculated based on the BoltzmannMatano analysis. The fitted profiles were also used in the determination of the impurity diffusion coefficients for $\mathrm{Al}$ and $\mathrm{Zn}$ in $\mathrm{Mg}$, calculated based on the Hall analysis. The activation energies and the preexponential factors for the composition-independent average effective interdiffusion coefficient and impurity diffusion coefficient were also calculated.
Table I. Diffusion anneal parameters.

\begin{tabular}{|c|c|c|}
\hline \multirow{2}{*}{ Couple } & $\begin{array}{c}\text { Temperature } \\
\left({ }^{\circ} \mathrm{C}\right)\end{array}$ & Time (hrs) \\
\hline \multirow{3}{*}{ Mg-MA9 } & 623 & 96 \\
\cline { 2 - 3 } & 673 & 17 \\
\cline { 2 - 3 } & 723 & 24 \\
\hline \multirow{3}{*}{ Mg-MZ6 } & 623 & 48 \\
\cline { 2 - 3 } & 673 & 8 \\
\cline { 2 - 3 } & 723 & 24 \\
\hline
\end{tabular}

\section{Calculation of Interdiffusion Coefficients}

Composition-dependent interdiffusion cofficient, $\widetilde{D}$, can be determined from concentration profiles using Fick's First Law expressed as:

$$
\tilde{J}=-\widetilde{D}_{l} \frac{\partial c_{i}}{\partial x}
$$

using the Boltzmann-Matano method. The first step is to find the Matano plane. The Matano plane is the graphically determined position in the concentration profile where there is a mass balance such that:

$$
\int_{C_{i}^{ \pm \infty}}^{C_{i}^{o}} x d C_{i}+\int_{C_{i}^{o}}^{C_{i}^{-\infty}} x d C_{i}=0
$$

where $C_{i}^{ \pm \infty}$ refers to the composition at the terminal ends of the diffusion couple, and $C_{i}^{o}$ refers to the composition at the Matano plane [6]. The interdiffusion flux, $\tilde{J}_{i}$ was calculated using:

$$
\tilde{J}_{i}=\frac{1}{2 t} \int_{C_{i}^{ \pm \infty}}^{C_{i}}\left(x-x_{o}\right) d C_{i}
$$

where $\mathrm{x}_{\mathrm{o}}$ is the location of the Matano plane. The interdiffusion coefficient, $\widetilde{D}_{i}$ was calculated by combining Eqs. (1) and (3) to yield:

$$
\widetilde{D}_{i}=\frac{\frac{1}{2 t} \int_{C_{i}^{ \pm \infty}}^{C_{i}}\left(x-x_{o}\right) d C_{i}}{\frac{\partial C_{i}}{\partial x}}
$$

\section{Calculation of Impurity Diffusion Coefficients}

The Boltzmann-Matano method can be unreliable at the compositional extremes of the profile, because the concentration gradient is difficult to determine as the composition approaches that of the terminal end. The Hall Method puts the concentration gradient in terms of a Gaussian probability distribution, thus 
permitting a more accurate determination of the interdiffusion coefficient at impurity levels. Using probability theory, Hall proposed that, since time is constant for a given experiment, a probability plot of the concentration distribution will yield a straight line whose slope and intercepts can be used to solve the concentration-dependent diffusion coefficient [7-8]. $\mathrm{C} / \mathrm{C}_{\infty}$ is a probability and thus written in terms of the cumulative standard normal density function or $\mathrm{C} / \mathrm{C}_{\infty}$ $=1 / 2+1 / 2$ erf $\mathrm{u}$ in which $\mathrm{u}=\mathrm{h} \eta+\mathrm{k}$ where $\mathrm{h}$ is the slope and $\mathrm{k}$ is the intercept of the straight line plot, and $\eta$ is the Boltzmann variable, $x / 2\left(t^{1 / 2}\right)$. Thus, the diffusion equation can be rewritten in terms of $\mathrm{h}, \mathrm{k}$, and u such that:

$$
D=\frac{1}{h^{2}}+\frac{k \sqrt{\pi}}{h^{2}}(1+\operatorname{erf} u) \exp \left(u^{2}\right)
$$

\section{Calculation of Activation Energy for Diffusion}

Solid-state diffusion is strongly dependent on temperature, and diffusion coefficients increase with increasing temperature. The temperature dependence of diffusion coefficients, in general, fits an Arrhenius model: a generalized relation between the interdiffusion coefficient and temperature.

$$
\widetilde{D}=\widetilde{D_{o}} \exp \left[-\frac{\widetilde{Q_{D}}}{R T}\right]
$$

$\widetilde{D}_{o}$ is the pre-exponential factor and $\widetilde{Q}_{D}$ is the activation energy for interdiffusion. Temperature is the absolute temperature and $\mathrm{R}$ is gas constant.

\section{Results and Discussion}

A typical measured solute concentration data and fitted concentration profile from diffusion couple $\mathrm{Mg}$ vs. MA9 annealed at $450^{\circ} \mathrm{C}$ for 24 hours is shown in Figure 2. The open circles represent EPMA data points while the solid line is the fitted concentration profile. It is evident in this representative profile the asymptotic regions do not mirror each other thus indicating a concentration dependence on the diffusivity.

From the fitted concentration profiles, the Boltzmann-Matano method was applied to determine the interdiffusion coefficient. Similarly, the interdiffusion coefficient was calculated for the dilute regime via the Hall analytical method. The interdiffusion coefficient as a function of composition for $\mathrm{Al}$ and $\mathrm{Zn}$ in $\mathrm{Mg}(\mathrm{ss})$ is presented in Figures 3 and 4, respectively. The interdiffusion coefficients on the left hand side of the dotted divisor has been calculated using the Hall method while those on the right hand side were determined from the Boltzmann-Matano approach, as indicated.

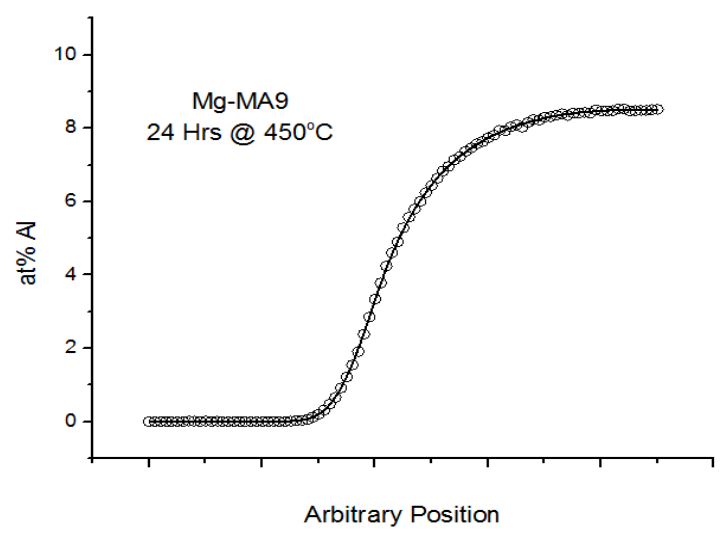

Figure 2: EPMA data and fitted concentration profile for the Mg-MA9 couple annealed for 24 hours at $450^{\circ} \mathrm{C}$.

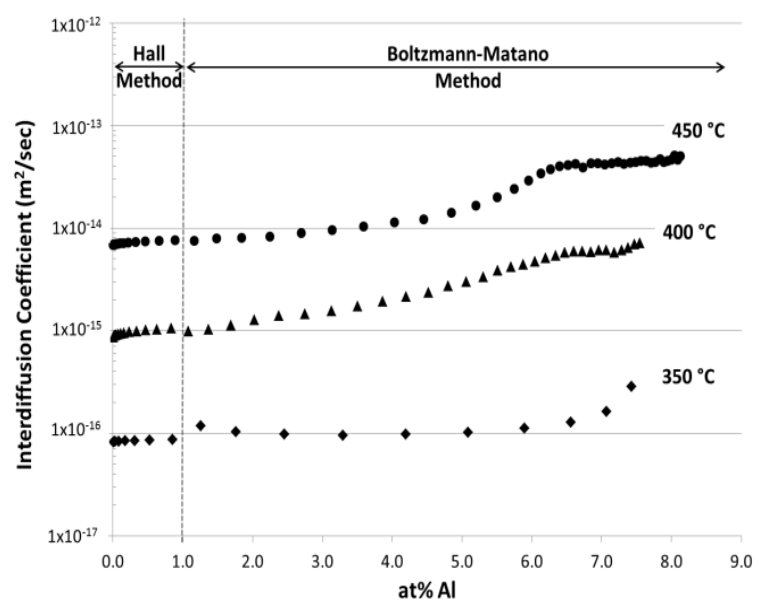

Figure 3: Interdiffusion coefficient as a function of $\mathrm{Al}$ in the $\mathrm{Mg}$ solid solution. The demarcation at 1 at.\% Al separates the analysis based on the Hall method and that based on the Boltzmann-Matano method.

The profile trends upward from left to right; this positive trend becomes more evident as the temperature increases. Since the addition of $\mathrm{Al}$ decreases the melting point of the $\operatorname{Mg}(\mathrm{ss})$, interdiffusion coefficients would be expected to increase with an increasing $\mathrm{Al}$ content. At the lowest temperature, the interdiffusion appears to be independent of concentration, remaining virtually constant throughout the examined compositional range. There is good agreement within the data for the two analysis methods. 


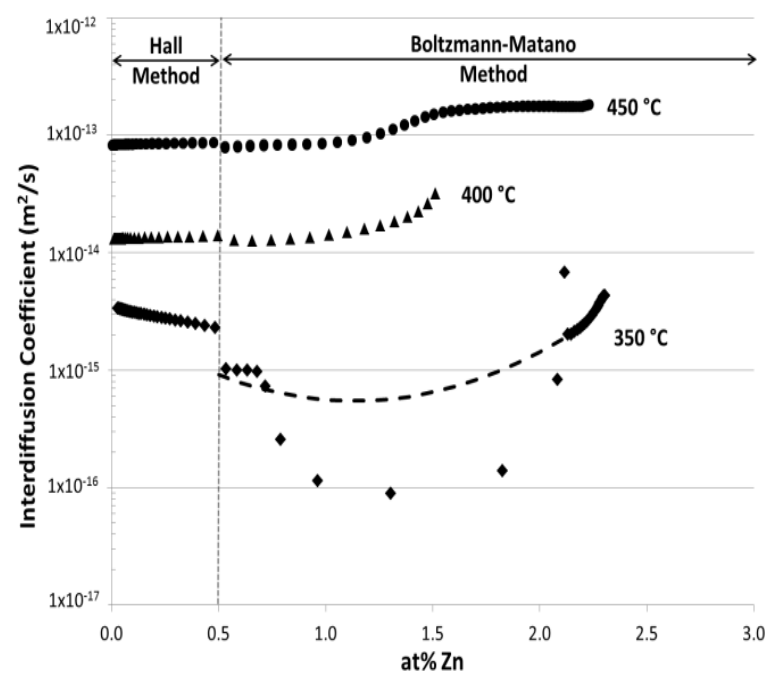

Figure 4: Interdiffusion coefficients as a function of $\mathrm{Zn}$ in the $\mathrm{Mg}$ solid solution. The vertical marker at 0.5 at. $\% \mathrm{Zn}$ delineates the analysis based on the Hall method and that based on the Boltzmann-Matano method.

Compared to the $\mathrm{Al}$, the interdiffusion in the $\mathrm{Mg}(\mathrm{ss})$ of the $\mathrm{Mg}-\mathrm{Zn}$ system is relatively unchanged over the composition range investigated. At the lowest temperature, the interdiffusion zone was very narrow and reliable compositional profiles were difficult to extract. Nonetheless, the Hall analytical method yields consistent data which can be used for the most dilute of alloys.

Using the Hall diffusion coefficient at the infinitely dilute composition, the activation energy and preexponential factor of the impurity diffusion can be determined. The pre-exponential factor and activation energy for $\mathrm{Al}$ impurity diffusion in $\mathrm{Mg}$ is $1.61 \times 10^{-4}$ $\mathrm{m}^{2} / \mathrm{sec}$ and $144.1 \mathrm{~kJ} / \mathrm{mol}$, respectively. For $\mathrm{Zn}$ in $\mathrm{Mg}$, the impurity diffusion pre-exponential factor and activation energy is $1.03 \times 10^{-5} \mathrm{~m}^{2} / \mathrm{sec}$ and 109.8 $\mathrm{kJ} / \mathrm{mol}$. Figure 5 presents the impurity diffusion coefficients determined in this study with those determine in other studies. Čermák used isotopic tracer techniques to determine the $\mathrm{Zn}$ impurity diffusivity [9]. Brennan ascertained the Al impurity diffusion coefficients through thin film depth profiling procedures [10]. Ganeshan calculated the impurity diffusion coefficients using first-principles calculations [11].

As can be seen in Figure 5, the Zn impurity diffusion is consistent with Čermák's findings. The congruency in results between the isotopic tracer diffusion coefficient and the impurity diffusion coefficient lends credence to the analytical method presented herein. Because it is monoisotipic, little data is available for Al impurity diffusion. Brennan's work, while ground-breaking, acknowledged that the refined microstructure and depth profiling methodology employed resulted in a broadening of the diffusion profile which led to somewhat inflated diffusion coefficients [10]. Thus, as expected, the Al impurity diffusion coefficients established in this study are slightly lower than previously published.

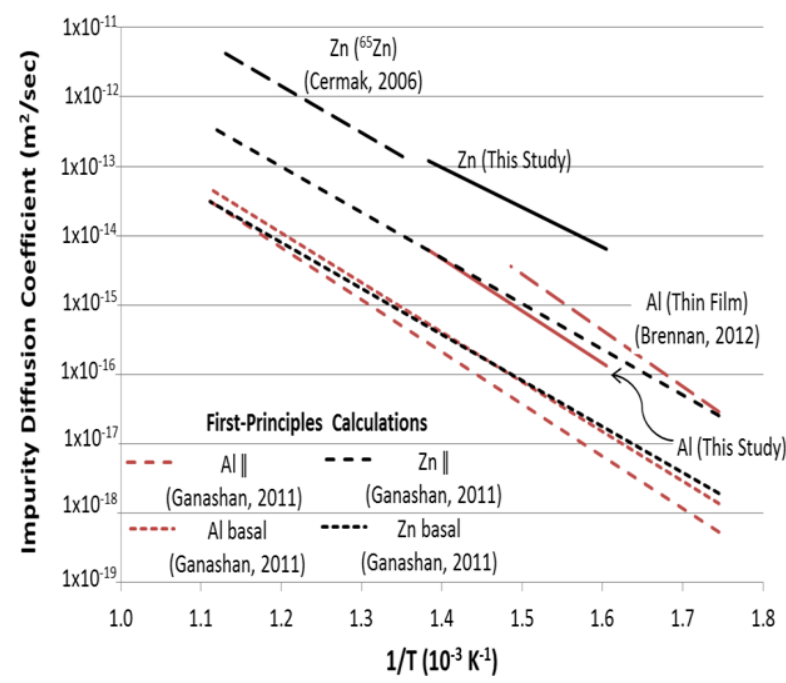

Figure 5: Comparison of $\mathrm{Al}$ and $\mathrm{Zn}$ impurity diffusion coefficients in $\mathrm{Mg}$ calculated by different methods.

Figure 6 presents the impurity diffusion coefficients determined in this study alongside those of other elements. As is customary, tracer diffusion studies where performed in the determination of $\mathrm{Mg}$ selfdiffusion coefficients and impurity diffusion coefficients for In and Fe. [12] The impurity diffusion coefficients for Ce and La were analytically determined by assuming the diffusivity to be constant and measuring the rate of precipitate dissolution. [13] Because $\mathrm{Al}$ in monoisotopic, In has been used as a substitute for $\mathrm{Al}$ in diffusion studies. The primary reason for this substitution is that In diffuses in $\mathrm{Al}$ at the same rate as Al self-diffusion [14]. However, examination of Figure 6 provides some indication that In impurities diffuses faster than $\mathrm{Al}$ impurities in $\mathrm{Mg}$, in particular at lower temperatures. The kinetic behavior of Be impurities, on the other hand, appears to be similar to that of $\mathrm{Al}$ impurities in $\mathrm{Mg}$. Further consideration of Figure 6 reveals $\mathrm{Zn}$ impurity diffusion to be faster than $\mathrm{Mg}$ self-diffusion which, in turn, is faster than the $\mathrm{Al}$ impurity diffusion. 


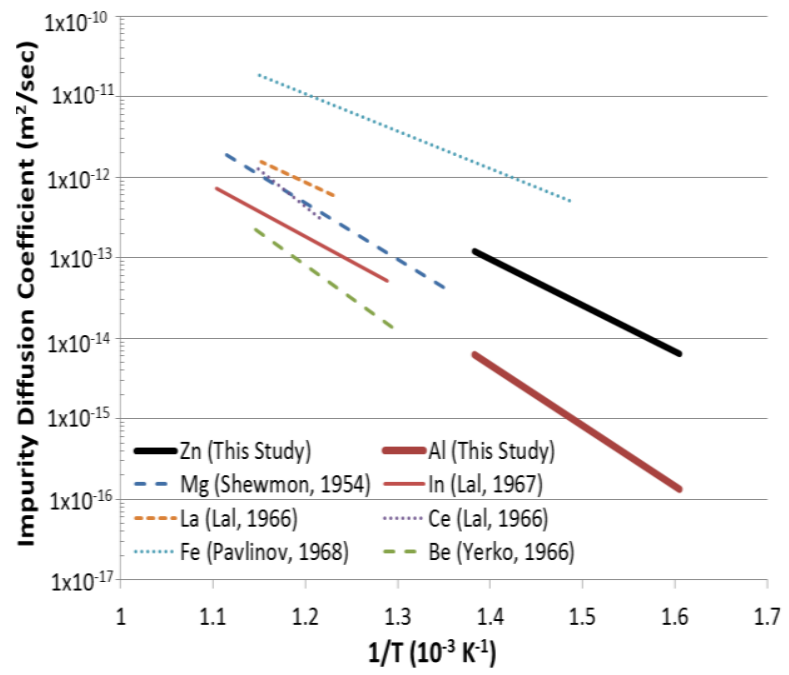

Figure 6: Comparison of $\mathrm{Al}$ and $\mathrm{Zn}$ impurity diffusion coefficients determined in this study with impurity diffusion coefficients for other elements in Mg.

\section{Conclusions}

Diffusion in the $\mathrm{Mg}$ rich solid solution region of the $\mathrm{Mg}-\mathrm{Al}$ and $\mathrm{Mg}-\mathrm{Zn}$ binary systems was investigated from $350{ }^{\circ} \mathrm{C}$ to $450{ }^{\circ} \mathrm{C}$. Through this study, the Hall analytical method has been shown to be an effective and reliable method for acquiring impurity diffusion data from solid-to-solid interdiffusion couples. The pre-exponential factor and activation energy for $\mathrm{Al}$ impurity diffusion in $\mathrm{Mg}$ is $1.61 \times 10^{-4} \mathrm{~m}^{2} / \mathrm{sec}$ and $144.1 \mathrm{~kJ} / \mathrm{mol}$, respectively. For $\mathrm{Zn}$ in $\mathrm{Mg}$, the impurity diffusion pre-exponential factor and activation energy is $1.03 \times 10^{-5} \mathrm{~m}^{2} / \mathrm{sec}$ and 109.8 $\mathrm{kJ} / \mathrm{mol}$. Zn migrates faster than $\mathrm{Mg}$ self-diffusion, while $\mathrm{Al}$ diffuses slower than $\mathrm{Mg}$ self-diffusion.

\section{Acknowledgement}

This research was sponsored by the U.S. Department of Energy, Assistant Secretary for Energy Efficiency and Renewable Energy, Office of Vehicle Technologies, as part of the Lightweight Materials Program.

\section{References}

[1] B.L. Mordike, T. Ebert, "Magnesium: Properties-applications-potential," Materials Science and Engineering A, 302, 2001, 37-45.

[2] A. Luo, "Magnesium: Current and Potential Automotive Applications," Journal of Materials, 54, 2002, 42-48.

[3] M.K. Kulekci, "Magnesium and its alloys applications in automotive industry,"
International Journal of Advanced Manufacturing Technology, 39, 2008, 851-865.

[4] R. Urbance, F. Field, R. Kirchain, R. Roth, J. Clark, "Market Model Simulation: The Impact of Increased Automotive Interest in Magnesium," Journal of Materials, 54, 2002, 25-33.

[5] A.K. Mondal, D. Fechner, S. Kumar, H. Dieringa, P. Maier, K.U. Kainer, "Interrupted creep behavior of $\mathrm{Mg}$ alloys developed for powertrain applications," Materials Science and Engineering A, 527, 2010, 2289-2296.

[6] C. Matano, Jrn. J. Phys., (1933), Vol. 8, pp. 109

[7] L. Hall, "An Analytical Method of Calculating Variable Diffusion Coefficients," Journal of Chemical Physics, 21, 1953, 87-89.

[8] N. Sarafianos, "An Analytical Method of Calculating Variable Diffusion Coefficients," Journal of Materials Science, 21, 1983, 22832288.

[9] J. Čermák, I. Stoukal, "Diffusion of ${ }^{65} \mathrm{Zn}$ in $\mathrm{Mg}$ and in Mg-xAl solid solutions," Phys. Stat. Sol., 203, 2006, 2386-2392.

[10] S. Brennan, A.P. Warren, K.R. Coffey, N. Kulkarni, P. Todd, M. Kilmov, Y.H. Sohn, "Aluminum Impurity Diffusion in Magnesium," Journal of Phase Equilibria and Diffusion, 33, 2012, 1547-7037.

[11]S. Ganeshan, L.G. Hector Jr., Z.K. Liu, "FirstPrinciples calculations of impurity diffusion coefficients in dilute $\mathrm{Mg}$ alloys using the 8frequency model," Acta Materialia, 59, 2011, 3214-3228.

[12] L.V. Pavlinov, A.M. Gladyshev, V.N. Bykov, Phys. Met. Metallogr, 26, 1968,

[13] K. Lal, Diffusion of Some Elements in Magnesium, CEA Report, 1967, R 3136, p 54

[14]M. Eggersmann, H. Mehrer, "Diffusion in intermetallic phases of the Fe-Al system," Philosophical Magazine A, 80:5, 2000, 12191244. 
